\title{
Robust Fault Diagnosis of Proton Exchange Membrane Fuel Cells using a Takagi-Sugeno Interval Observer Approach
}

\author{
Damiano Rotondo a,*, Rosa M. Fernandez-Canti ${ }^{\mathrm{a}}$, Sebastian Tornil-Sin ${ }^{\mathrm{a}}$, \\ Joaquim Blesa ${ }^{a, b}$, Vicenç Puiga,b \\ ${ }^{a}$ Department of Automatic Control (ESAII), Technical University of Catalonia (UPC), \\ Rambla de Sant Nebridi 10, 08222 Terrassa (Spain). Tel: +34 937398973 \\ ${ }^{b}$ Institute of Robotics and Industrial Informatics (IRI), UPC-CSIC, Carrer de Llorens $i$ \\ Artigas, 4-6, 08028 Barcelona (Spain)
}

\section{Abstract}

In this paper, the problem of robust fault diagnosis of proton exchange membrane (PEM) fuel cells is addressed by introducing the Takagi-Sugeno (TS) interval observers that consider uncertainty in a bounded context, adapting TS observers to the so-called interval approach. Design conditions for the TS interval observer based on regional pole placement are also introduced to guarantee the fault detection and isolation (FDI) performance. The fault detection test is based on checking the consistency between the measurements and the output estimations provided by the TS observers. In presence of bounded uncertainty, this check relies on determining if all the measure-

\footnotetext{
${ }^{*}$ Corresponding author

Email address: damiano.rotondo@yahoo.it (Damiano Rotondo)
} 
ments lie inside their corresponding estimated interval bounds. When a fault is detected, the measurements that are inconsistent with their corresponding estimations are annotated and a fault isolation procedure is triggered. By using the theoretical fault signature matrix (FSM), which summarizes the effects of the different faults on the available residuals, the fault is isolated by means of a logic reasoning that takes into account the bounded uncertainty, and if the number of candidate faults is more than one, a correlation analysis is used to obtain the most likely fault candidate. Finally, the proposed approach is tested using a PEM fuel cell case study proposed in the literature.

Keywords: PEM fuel cells, model-based fault diagnosis, Takagi-Sugeno fuzzy systems, analytical redundancy relations, interval observers.

\section{Introduction}

In the last years, major efforts to reduce greenhouse effects and pollution have increased the demand for environmentally friendlier energy sources [1]. Fuel cells have been pointed out as a promising alternative way for an energy source in the future, not only because they are considered a zero-emission power source, but also because they are very quite, and reduce noise pol-

lution. Their energy density makes them specially suitable for embedded 
generation systems in transport applications. Proton exchange membrane fuel cells (PEMFC) are electrochemical devices in which the energy of a reaction between a fuel, the hydrogen, and an oxidant, the oxygen, is directly and continuously converted into electrical energy, obtaining water as a subproduct [2]. Many control strategies for PEMFC have been proposed in the recent literature, e.g. optimal control [3], model predictive control [4] and sliding mode control [5].

However, fuel cell technology is still too expensive to be accessible to a mass market, and there are still considerable difficulties to overcome. One of the main drawbacks of fuel cell systems is related to the stack lifetime and reliability when working at strongly changing charge conditions, like those that could be usually found in transport applications. Several papers have analyzed the PEMFC durability taking into account the phenomena that could affect the PEMFC lifetime $[6,7,8]$, e.g. catalyst degradation, fuel starvation or inadequate water management $[9,10,11]$. To overcome these drawbacks, adequate fault detection and isolation (FDI) and fault-tolerant control (FTC) strategies are needed to avoid failure modes which could compromise the stack reliability and integrity. Real-time fault diagnosis (detection and isolation) can provide valuable information either for monitoring 
PEMFC operation, allowing to reconfigure/accommodate the control loop whenever any malfunction is reported, or to suggest preventive maintenance actions, to extend the lifetime of the system and thereby avoiding future damage in the equipment.

In [12], fault diagnosis of PEMFC is reviewed with a special emphasis on model-based methods. Fuel cells are complex and strongly non-linear systems in which continuously varying parameters are difficult to identify, even for static operation modes. Different authors have chosen different approaches for fuel cell system modeling. Electric equivalent models [13], state space models $[14,15]$ and bond graph representation models [16] are the most representative approaches. Even if these models can represent fuel cell behavior at some operating conditions, their application to fault diagnosis is not so straightforward and, moreover, most of them are related just to the stack and not to the overall system.

Recently, the complex and non-linear dynamics of the power generation systems based on fuel cell technology, described in detail in [14], led to the use of linear models that include parameters varying with the operating point (known as linear parameter varying or LPV models) not only for advanced control [17] but also for model-based fault diagnosis [18] purposes. The use of 
Takagi-Sugeno (TS) models [19] is an alternative to LPV models as a linearlike modeling framework that allows extending control [20] and FDI methods to non-linear systems [21]. Thus, this paper will consider TS models and observers for FDI in PEM fuel cells. In the literature, the use of the TS has been widely considered for FDI (see, e.g. [21, 22]) and fault estimation/FTC (see e.g. [23, 24]). However, due to the presence of parametric uncertainties in the TS model, the design of a conventional observer that converges to the exact value of the state is complicated [25]. In this situation, an interval estimation is still possible, and an observer that evaluates the set of admissible values for the state at each time instant can be designed [25, 26, 27, 28, 29]. The main contribution of this paper is to address the problem of robust fault diagnosis of PEM fuel cells by introducing the TS interval observers that consider uncertainty in a bounded context, adapting TS observers to the socalled interval approach. Design conditions for the TS interval observer based on regional pole placement are also introduced to guarantee the FDI performance. A bank of observers is designed by applying the structural analysis [30] to the PEMFC model considering the set of available sensors. The fault detection test is based on checking the full consistency between the measurements and the output estimations provided by the TS interval observers. In 
presence of bounded uncertainty, this check relies on determining if all the measurements lie inside their corresponding estimated interval bounds. In the case a fault is detected, the measurements that are inconsistent with their corresponding estimations are annotated and a fault isolation procedure is triggered. By using the theoretical fault signature matrix (FSM), the fault is isolated by means of a logic reasoning that takes into account the presence of bounded uncertainty, and a posterior correlation analysis provides the most likely fault candidate. Finally, the proposed approach is tested using a well known PEMFC case study proposed in the literature in [14] by deriving an interval TS model and observer.

The structure of the paper is the following. Section 2 describes the PEMFC case study and presents its linear-like state-space model. Section 3 introduces the TS interval observer approach. Section 4 presents the proposed fault diagnosis methodology based on TS interval observers. Then, Section 5 presents the application of the proposed fault diagnosis approach to the considered PEMFC case study. Finally, Section 6 gives the main conclusions. 


\section{Modeling of the PEMFC}

\subsection{Description}

The fault diagnosis approach proposed in this paper is tested on a typical PEMFC system (see Fig. 1) based on the model provided in [2].

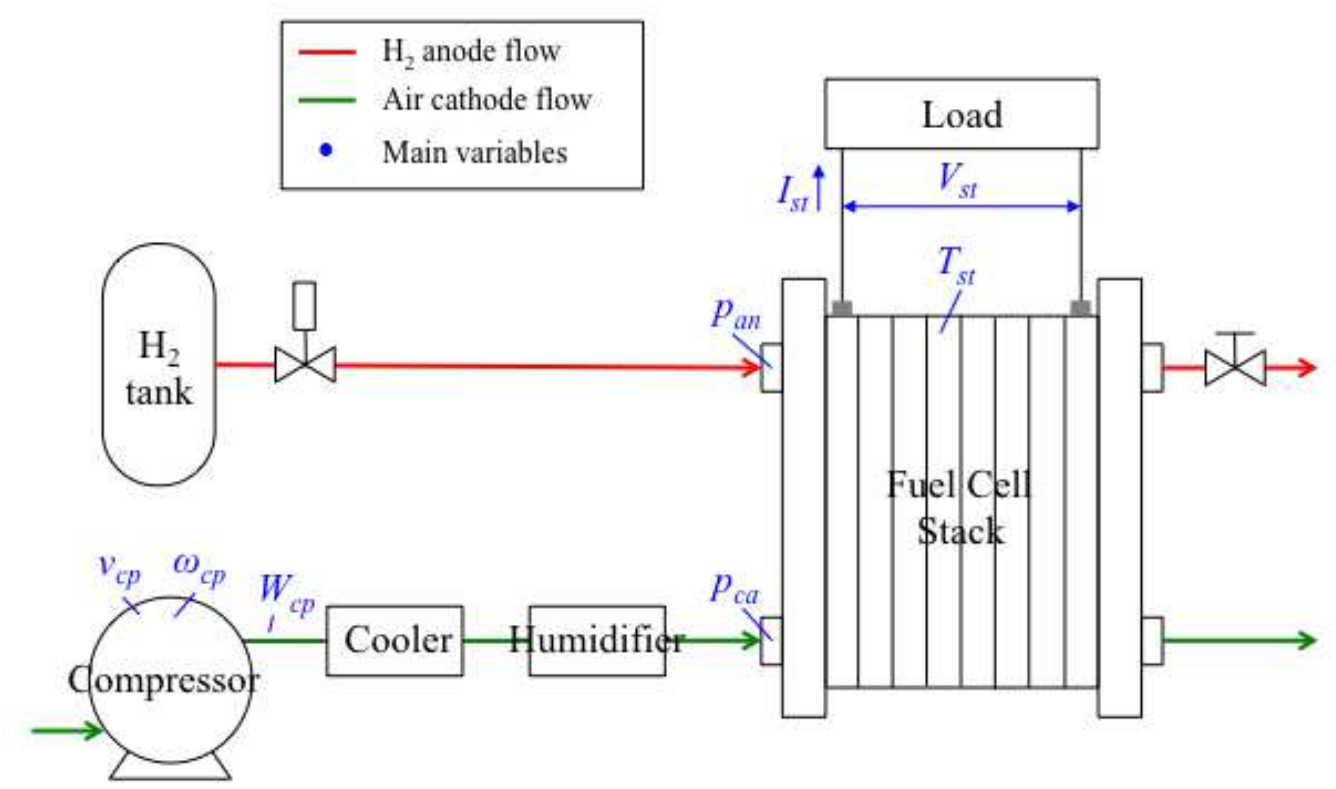

Figure 1: PEM fuel cell system.

The fuel cell, in response to a current demand by an electrical load, consumes oxygen and hydrogen and generates water and heat. The hydrogen is provided by the hydrogen supply system, whose main components are a pressurized hydrogen tank and a supply servo valve that allows controlling the hydrogen flow or pressure. The air supply system, consisting mainly of a 
compressor driven by an electric motor, provides the air flow. The control of the hydrogen and air supply systems aims at maintaining the required partial pressures of the hydrogen and air entering into the anode and the cathode of the stack, respectively. Additionally, since the compressor provides air at a high temperature due to the increased pressure, the cooling system is used to reduce the temperature of the air entering into the stack in order to prevent the fuel cell membrane from damaging. Finally, a humidifier acts on the cathode path to prevent membrane damage due to dehydration.

The main variables involved in the PEMFC system operation are listed in Table 1.

\begin{tabular}{ll}
\hline Symbol & Description \\
\hline$v_{c p}$ & Compressor input voltage \\
$\omega_{c p}$ & Compressor speed \\
$W_{c p}$ & Compressor air flow \\
$p_{c a}$ & Air pressure in the cathode \\
$p_{a n}$ & Hydrogen pressure in the anode \\
$T_{s t}$ & Stack temperature \\
$I_{s t}$ & Stack current \\
$v_{s t}$ & Stack voltage \\
\hline
\end{tabular}

Table 1: PEMFC variables.

Following other works in the literature, e.g. [31], six variables are assumed to be measured:

- Compressor speed $\left(\omega_{c p}\right)$. 
- Pressure in the cathode supply manifold $\left(p_{s m}\right)$.

- Mass of hydrogen in the anode $\left(m_{H_{2}}\right)$.

- Pressure in the anode $\left(p_{a n}\right)$.

- Pressure in the cathode $\left(p_{c a}\right)$.

- Stack voltage $\left(v_{s t}\right)$.

The feedback control and safe operation of a PEMFC can be compromised by sensor faults. Hence, it is important to develop fault diagnosis methodologies that are able to detect and isolate such faults.

In this paper, faults affecting the previously listed sensors are considered. These faults will be identified as $f_{1}, f_{2}, \ldots, f_{6}$, in accordance with the order provided in the list. The extension to other types of faults is straightforward by applying the proposed methodology to the new set of faults.

\subsection{Linear-like representation of the PEMFC model}

The model used in this paper is derived from the non-linear equations presented in [14], that are valid under the following assumptions: the stack temperature $T_{s t}$ is constant (it changes slowly with respect to the other system variables); the temperature and humidity of the inlet reactant flows are 
perfectly controlled; the cathode and anode volumes of the multiple fuel cells are lumped as a single stack cathode and anode volumes; all the reactants behave as ideal gases.

According to [14], the stack current $I_{s t}$ can be considered as the system input and nine state variables can be used to describe the system dynamics in a non-linear state space representation: mass of oxygen in the cathode $\left(m_{\mathrm{O}_{2}}\right)$, mass of hydrogen in the anode $\left(m_{H_{2}}\right)$, mass of nitrogen in the cathode $\left(m_{N_{2}}\right)$, compressor speed $\left(\omega_{c p}\right)$, air pressure in the supply manifold $\left(p_{s m}\right)$, air mass in the supply manifold $\left(m_{s m}\right)$, mass of water in the anode $\left(m_{w, a n}\right)$, mass of water in the cathode $\left(m_{w, c a}\right)$, and pressure in the return manifold $\left(p_{r m}\right)$.

The non-linear model of the PEMFC can be reshaped into a linear-like 
representation, obtaining a model with the following structure:

$$
\left.\begin{array}{c}
\left(\begin{array}{c}
\dot{m}_{O_{2}} \\
\dot{m}_{H_{2}} \\
\dot{m}_{N_{2}} \\
\dot{m}_{s m} \\
\dot{p}_{s m} \\
\dot{p}_{r m} \\
\dot{\omega}_{c p} \\
\dot{m}_{w, a n} \\
\dot{m}_{w, c a}
\end{array}\right]=\left[\begin{array}{cccccccccc}
\tilde{\alpha}_{11} & 0 & \tilde{\alpha}_{13} & 0 & \alpha_{15} & \tilde{\alpha}_{16} & 0 & 0 & 0 \\
0 & \tilde{\alpha}_{22} & 0 & 0 & \tilde{\alpha}_{25} & 0 & 0 & \tilde{\alpha}_{28} & 0 \\
\tilde{\alpha}_{31} & 0 & \tilde{\alpha}_{33} & 0 & \alpha_{35} & \tilde{\alpha}_{36} & 0 & 0 & 0 \\
\alpha_{41} & 0 & \alpha_{43} & 0 & \alpha_{45} & 0 & 0 & 0 & 0 \\
\tilde{\alpha}_{51} & 0 & \tilde{\alpha}_{53} & 0 & \tilde{\alpha}_{55} & 0 & 0 & 0 & 0 \\
\alpha_{61} & 0 & \alpha_{63} & 0 & 0 & \tilde{\alpha}_{66} & 0 & 0 & 0 \\
0 & 0 & 0 & 0 & 0 & 0 & \tilde{\alpha}_{77} & 0 & 0 \\
0 & \tilde{\alpha}_{82} & 0 & 0 & \tilde{\alpha}_{85} & 0 & 0 & \tilde{\alpha}_{88} & 0 \\
\tilde{\alpha}_{91} & 0 & \tilde{\alpha}_{93} & 0 & \tilde{\alpha}_{95} & \tilde{\alpha}_{96} & 0 & 0 & 0
\end{array}\right]\left(\begin{array}{c}
m_{O_{2}} \\
m_{H_{2}} \\
m_{N_{2}} \\
m_{s m} \\
p_{s m} \\
p_{r m} \\
\omega_{c p} \\
m_{w, a n} \\
m_{w, c a}
\end{array}\right) \\
+\left(\begin{array}{ccccccccccc}
\tilde{\gamma}_{1} & \tilde{\gamma}_{2} & \tilde{\gamma}_{3} & \tilde{\gamma}_{4} & \tilde{\gamma}_{5} & \gamma_{6} & \tilde{\gamma}_{7} & \tilde{\gamma}_{8} & \tilde{\gamma}_{9}
\end{array}\right)^{T} \\
\beta_{1} \beta_{2} \\
0
\end{array}\right]
$$

where the coefficients with tilde, e.g. $\tilde{\alpha}_{11}$, are functions of the scheduling parameter $I_{s t}$ and the remaining are constant. All the coefficients depend on constants (physical and chemical constants, physical coefficients related with the PEMFC construction and operating conditions) whose values are taken from [14].

The measured variables $\omega_{c p}, p_{s m}$ and $m_{H_{2}}$ correspond directly to state 
variables in (1). On the other hand, $p_{a n}, p_{c a}$ and $v_{s t}$ are related to some of the state variables according to the following general expressions:

$$
\begin{aligned}
& p_{c a}=f\left(m_{\mathrm{O}_{2}}, m_{N_{2}}\right) \\
& p_{a n}=f\left(m_{H_{2}}, m_{w, a n}\right) \\
& v_{s t}=f\left(m_{H_{2}}, m_{O_{2}}, p_{c a}\right)
\end{aligned}
$$

\section{Takagi-Sugeno interval observers}

\subsection{System representation}

TS systems, as proposed in [19], are described by local models merged together using IF-THEN rules [32]. In particular, in this paper, we will consider an uncertain discrete-time TS system, as follows:

$$
\begin{aligned}
& \operatorname{IF} \vartheta_{1}(k) \text { is } M_{i 1} A N D \ldots A N D \vartheta_{p}(k) \text { is } M_{i p} \\
& \operatorname{THEN}\left\{\begin{array}{l}
x_{i}(k+1)=\left(A_{0 i}+\Delta A_{i}\right) x(k)+B_{i} u(k)+d(k) \\
y_{i}(k)=C x(k)+v(k)
\end{array}\right.
\end{aligned}
$$

where $x(k) \in \mathbb{R}^{n_{x}}$ is the state vector, $u(k) \in \mathbb{R}^{n_{u}}$ is the input vector, $y(k) \in \mathbb{R}^{n_{y}}$ is the output vector available for measurements, $d(k) \in \mathbb{R}^{n_{x}}$ is the exogenous disturbance and $v(k) \in \mathbb{R}^{n_{y}}$ is the measurement noise. $M_{i j}$ 
denote the fuzzy sets and $N$ is the number of model rules. $\vartheta_{1}(k), \ldots, \vartheta_{p}(k)$ are the premise variables that can be functions of endogenous and/or exogenous variables. Each linear consequent equation is called a subsystem.

Given $x(k), u(k), d(k)$ and $v(k)$, the state of the TS system at time $k+1$ and the output at time $k$ can be easily inferred as:

$$
\begin{gathered}
x(k+1)=\sum_{i=1}^{N} \rho_{i}(\vartheta(k))\left[\left(A_{0 i}+\Delta A_{i}\right) x(k)+B_{i} u(k)+d(k)\right] \\
y(k)=C x(k)+v(k)
\end{gathered}
$$

where $\vartheta(k)=\left[\vartheta_{1}(k), \ldots, \vartheta_{p}(k)\right]^{T}$ is the vector containing the premise variables, and $\rho_{i}(\vartheta(k))$ is defined as follows:

$$
\rho_{i}(\vartheta(k))=\frac{\prod_{j=1}^{p} M_{i j}\left(\vartheta_{j}(k)\right)}{\sum_{i=1}^{N} \prod_{j=1}^{p} M_{i j}\left(\vartheta_{j}(k)\right)}
$$

where $M_{i j}\left(\vartheta_{j}(k)\right)$ is the grade of membership of $\vartheta_{j}(k)$ in $M_{i j}$ and $\rho_{i}(\vartheta(k))$ is such that:

$$
\left\{\begin{array}{l}
\sum_{i=1}^{N} \rho_{i}(\vartheta(k))=1 \\
\rho_{i}(\vartheta(k)) \geq 0, \quad i=1, \ldots, N
\end{array}\right.
$$

The matrices $A_{0 i} \in \mathbb{R}^{n_{x} \times n_{x}}, B_{i} \in \mathbb{R}^{n_{x} \times n_{u}}$ and $C \in \mathbb{R}^{n_{y} \times n_{x}}$ are known, 
whereas the matrices $\Delta A_{i} \in \mathbb{R}^{n_{x} \times n_{x}}$ represent the uncertainty, and are not known. Also, the exact values of $d(k)$ and $v(k)$ are not available. However, it is assumed that some bounds are known, as follows:

$$
\begin{gathered}
\underline{d}(k) \leq d(k) \leq \bar{d}(k) \\
|v(k)| \leq V(k) \\
\underline{\Delta A_{i}} \leq \Delta A_{i} \leq \overline{\Delta A_{i}}
\end{gathered}
$$

for some known functions $\underline{d}(k), \bar{d}(k), V(k)$ and for some known matrices $\underline{\Delta A_{i}}, \overline{\Delta A_{i}} \in \mathbb{R}^{n_{x} \times n_{x}}$. The relations $\leq$ are understood elementwise.

The fault diagnosis test is based on checking whether the measurements are consistent with the system model or not. However, due to the presence of uncertainty, unknown disturbances and noise, an exact estimation $\hat{x}(k)$ of the state $x(k)$, to be used for comparison with the measurements, cannot be obtained. However, taking into account (10)-(12), it is possible to design an observer that provides an interval estimation of $x(k)$, i.e. some lower and upper estimates $\underline{x}(k), \bar{x}(k)$ of $x(k)$, such that:

$$
\underline{x}(k) \leq x(k) \leq \bar{x}(k) \quad \forall k \in \mathbb{Z}
$$


This observer will be denoted as TS interval observer.

\subsection{The TS interval observer}

The TS interval observer for the system (5) has the following form, obtained as a slight modification of the one presented in [25]:

$$
\begin{aligned}
& I F \vartheta_{1}(k) \text { is } M_{i 1} A N D \ldots A N D \vartheta_{p}(k) \text { is } M_{i p} \\
& \qquad \begin{array}{r}
\underline{x_{i}}(k+1)=\left[A_{0 i}-\underline{L_{i}} C\right] \underline{x}(k)+B_{i} u(k) \\
+\underline{\Delta A_{i}}{ }^{+} \underline{x}^{+}(k)-{\overline{\Delta A_{i}}}^{+} \underline{x}^{-}(k) \\
-\underline{\Delta A_{i}}{ }^{-} \bar{x}^{+}(k)+{\overline{\Delta A_{i}}}^{-} \bar{x}^{-}(k) \\
+\underline{L_{i}} y(k)-\left|\underline{L_{i}}\right| V(k) E_{n_{y}}+\underline{d}(k) \\
\overline{x_{i}}(k+1)=\left[A_{0 i}-\overline{L_{i}} C\right] \bar{x}(k)+B_{i} u(k) \\
+\overline{\Delta A}^{+} \bar{x}^{+}(k)-\underline{\Delta A_{i}}{ }^{+} \bar{x}^{-}(k) \\
-{\overline{\Delta A_{i}}}^{-} \underline{x}^{+}(k)+\underline{\Delta A_{i}}{ }^{-} \underline{x}^{-}(k) \\
+\bar{L}_{i} y(k)+\left|\overline{L_{i}}\right| V(k) E_{n_{y}}+\bar{d}(k)
\end{array}
\end{aligned}
$$

where $\underline{L_{i}} \in \mathbb{R}^{n_{x} \times n_{y}}$ and $\overline{L_{i}} \in \mathbb{R}^{n_{x} \times n_{y}}$ are the observer gains to be designed,

${\overline{\Delta A_{i}}}^{+}=\max \left\{0, \overline{\Delta A_{i}}\right\},{\overline{\Delta A_{i}}}^{-}={\overline{\Delta A_{i}}}^{+}-\overline{\Delta A_{i}}, \underline{\Delta A_{i}}{ }^{+}=\max \left\{0, \underline{\Delta A_{i}}\right\}$, ${\underline{\Delta A_{i}}}^{-}=\underline{\Delta A_{i}}{ }^{+}-\underline{\Delta A_{i}}, \bar{x}^{+}=\max \{0, \bar{x}\}, \bar{x}^{-}=\bar{x}^{+}-\bar{x}, \underline{x}^{+}=\max \{0, \underline{x}\}$, $\underline{x}^{-}=\underline{x}^{+}-\underline{x}$ and, finally, $E_{n_{y}} \in \mathbb{R}^{n_{y} \times 1}$ denotes the column vector with all 
elements equal to 1 .

Then, the lower and upper estimates of $y(k)$ can be obtained as:

$$
\begin{aligned}
& \underline{y}(k)=C^{+} \underline{x}(k)-C^{-} \bar{x}(k) \\
& \bar{y}(k)=C^{+} \bar{x}(k)-C^{-} \underline{x}(k)
\end{aligned}
$$

where $C^{+}=\max \{0, C\}$ and $C^{-}=C^{+}-C$.

\subsection{Design conditions}

In order to guarantee a proper dynamics regarding fault detection, the interval estimation of $x(k)$ with constraints on the closed-loop poles location of the interval observer is desired. Although from the mathematical R1-3 point of view the concept of poles refers to linear time invariant (LTI) systems, [33] has shown that when pole placement in linear matrix inequality (LMI) regions [34] is considered, some transient properties related to the poles location, e.g. decay ratio, would also hold for the case of fuzzy TS systems. As discussed in [35], the observer poles play an important role in the observer fault sensitivity. The following theorem, based on the reasoning made by [25], provides some conditions to 
accomplish this goal under the assumption that:

$$
\underline{x}(0) \leq x(0) \leq \bar{x}(0)
$$

Theorem 1. Given an LMI region, defined as [34]:

$$
\mathcal{D}=\left\{z \in \mathbb{C}: f_{\mathcal{D}}(z)<0\right\}
$$

where the characteristic function $f_{\mathcal{D}}(z)$ is defined as:

$$
f_{\mathcal{D}}(z)=\alpha+z \beta+z^{*} \beta^{T}=\left\{\alpha_{k l}+\beta_{k l} z+\beta_{l k} z^{*}\right\}_{k, l \in[1, m]}
$$

with $\alpha=\alpha^{T} \in \mathbb{R}^{m \times m}$ and $\beta \in \mathbb{R}^{m \times m}$, if there exist a diagonal matrix $P \in \mathbb{R}^{2 n_{x} \times 2 n_{x}}$, a symmetric matrix $Q=Q^{T} \in \mathbb{R}^{2 n_{x} \times 2 n_{x}}$, block diagonal matrices $W_{i} \in \mathbb{R}^{2 n_{x} \times 2 n_{u}}, i=1, \ldots, N$ with the following structure:

$$
W_{i}=\left(\begin{array}{cc}
W_{i} \in \mathbb{R}^{n_{x} \times n_{u}} & 0 \\
0 & \overline{W_{i}} \in \mathbb{R}^{n_{x} \times n_{u}}
\end{array}\right)
$$

and constants $\varepsilon_{1}>0, \varepsilon_{2}>0, \gamma>0$ such that:

$$
\begin{aligned}
& P>0 \\
& Q>0
\end{aligned}
$$

and, for $i=1, \ldots, N$ :

$$
\begin{aligned}
& \left(\begin{array}{ccc}
\frac{P}{1+\varepsilon_{1}} & P D_{i}-W_{i} \Upsilon & \frac{P}{1+\varepsilon_{1}} \\
\left(P D_{i}-W_{i} \Upsilon\right)^{T} & P-Q-\gamma \eta^{2} I_{2 n_{x}} & 0 \\
\frac{P}{1+\varepsilon_{1}} & 0 & \gamma I_{2 n_{x}}-\varepsilon P
\end{array}\right) \geq 0 \\
& P\left[\begin{array}{cc}
A_{0, i} & 0 \\
0 & A_{0, i}
\end{array}\right]-W_{i} \Upsilon \geq 0 \\
& \left\{\alpha_{k l} P+\beta_{k l}\left(\left(\begin{array}{cc}
A_{0, i}^{T} & 0 \\
0 & A_{0, i}^{T}
\end{array}\right) P-\Upsilon^{T} W_{i}^{T}\right)\right. \\
& \left.+\beta_{l k}\left(P\left(\begin{array}{cc}
A_{0, i} & 0 \\
0 & A_{0, i}
\end{array}\right)-W_{i} \Upsilon\right)\right\}_{k, l \in[1, m]}<0
\end{aligned}
$$


with:

$$
\begin{gathered}
D_{i}=\left(\begin{array}{cc}
A_{0, i}+\underline{\Delta A_{i}^{+}} & 0 \\
0 & A_{0, i}+{\overline{\Delta A_{i}}}^{+}
\end{array}\right) \\
\Upsilon=\left(\begin{array}{cc}
C & 0 \\
0 & C
\end{array}\right) \\
\eta=2 \max _{i=1, \ldots, N}\left(\left\|{\underline{\Delta A_{i}}}^{+}-{\overline{\Delta A_{i}}}^{+}\right\|_{2}+\left\|\underline{\underline{\Delta A_{i}}}\right\|_{2}+\left\|{\overline{\Delta A_{i}}}^{-}\right\|_{2}\right) \\
\varepsilon=1+\varepsilon_{2}+\left(1+\varepsilon_{1}\right)^{-1}
\end{gathered}
$$

then, the TS interval observer (14) with gains calculated as:

$$
\begin{aligned}
& \underline{L_{i}}=P^{-1} \underline{W_{i}} \\
& \overline{L_{i}}=P^{-1} \overline{W_{i}}
\end{aligned}
$$

ensures the interval estimation of $x(k)$ given by (13), provided that (10)-(12) and (17) hold, and that:

$$
\exists ! i \in\{1, \ldots, N\} \mid \rho_{i}(\vartheta(k))=1, \rho_{j}(\vartheta(k))=0 \forall j \neq i, \forall k \in \mathbb{Z}
$$

Moreover, for each subsystem, the closed-loop poles of the TS interval observer are in $\mathcal{D}$.

Proof: See Appendix A.

The overall system of LMIs (21)-(24) and (25) can be solved efficiently using available solvers, e.g. the SeDuMi [36] in the YALMIP toolbox [37].

It is worth remarking that the proposed TS interval observer op- R1-2 erates under the assumption that the premise variables are known. Cases in which such assumption does not hold could be dealt with using results about TS observers with unmeasurable premise variables, see e.g. [38], but this extension goes beyond the goal of this 
paper.

\section{Fault diagnosis}

\subsection{Overview of the proposed fault diagnosis methodology}

The proposed fault diagnosis methodology uses a bank of TS interval observers, as described in Section 3, to take into account the uncertainty, and can be considered an alternative to the single observer methodology presented in $[18,39]$. By means of structural analysis, each observer can be made sensitive to a subset of faults, thus avoiding the need to perform a complex sensitive analysis, like the one in [39].

The whole procedure can be divided into off-line and on-line stages, and it can be summarized as follows.

\subsubsection{Off-line stage}

The linear-like representation of the PEMFC model provided in Section 2.2 is used as a starting point to obtain a set of analytical redundancy relations (ARRs) by means of structural analysis [30]. ARRs are equations relating only measured variables (so they can be evaluated on-line), that are satisfied in absence of faults. Each ARR is converted into a TS model by choosing the premise variables, and by considering a set of operating points 
that cover the whole range of system operation. Then, the model uncertainty associated to the TS models is adjusted in such a way that the outputs of the TS interval observers designed using these models "cover", with minimal over-bounding, all the measurements obtained in a complete fault-free scenario. This assures that no false alarms will occur when these TS interval observers are used for fault detection purposes.

\subsubsection{On-line stage}

At each time-instant, the TS observers provide estimations of the bounds of the measured variables, which would be compatible with the fault-free operation. The differences between the measurements and these bounds are called residuals, and are used for fault detection and isolation, as detailed in the following.

\subsection{Fault detection}

Fault detection is based on generating residuals, i.e. on comparing the measurements of the physical variables of the process $y(k)$ with the estimations $\hat{y}(k)$ provided by the associated system model:

$$
r(k)=y(k)-\hat{y}(k)
$$


where $r(k) \in \mathbb{R}^{n_{y}}$ is the residual vector.

When considering model uncertainty, the residual generated by (33) deviates from zero even in a non-faulty scenario. To cope with the parameter uncertainty effect, a passive robust approach based on adaptive thresholding can be used [40]. Thus, using this passive approach, the effect of the parameter uncertainty on the residual $r(k)$ associated to each system output $y(k)$ is bounded by the interval that will include the zero value in absence of faults. Hence, a fault detection test can be formulated as:

$$
0 \in[\underline{r}(k), \bar{r}(k)]
$$

where:

$$
\begin{aligned}
& \underline{r}(k)=y(k)-\bar{y}(k) \\
& \bar{r}(k)=y(k)-\underline{y}(k)
\end{aligned}
$$

being $y(k)$ the output coming from the sensors, $\underline{y}(k)$ and $\bar{y}(k)$ the bounds of the predicted output given by (15)-(16).

The fault detection test (34) can be alternatively formulated as:

$$
y(k) \in[\underline{y}(k), \bar{y}(k)]
$$




\subsection{Fault isolation}

Fault isolation consists in identifying the faults affecting the system. It is carried out on the basis of fault signatures, generated by the fault detection module, and their relation with all the considered faults. Robust residual evaluation presented in Section 4.2 allows obtaining a set of fault signatures $\phi(k)=\left[\phi_{1}(k), \phi_{2}(k), \ldots, \phi_{n_{y}}(k)\right]$, where each fault indicator is given by:

$$
\phi_{i}(k)=\left\{\begin{array}{ccc}
0 & \text { if } & 0 \in\left[\underline{r}_{i}(k), \bar{r}_{i}(k)\right] \\
1 & \text { if } & 0 \notin\left[\underline{r}_{i}(k), \bar{r}_{i}(k)\right]
\end{array}\right.
$$

The values of the fault signatures are compared with the theoretical FSM, denoted as $M$. An element $m_{i, j}$ ( $i$ indicates rows, $j$ indicates columns) of $M$ is equal to 1 if the fault $f^{j}$ affects the computation of the residual $r_{i}$; otherwise, the element $m_{i, j}$ is zero-valued.

Due to the uncertainty and to the different dynamics of the residuals, in presence of a given fault, the different fault indicators $\phi_{i}(k)$ that are sensitive to this particular fault can become active for non-overlapping short periods of time. In this case, proper fault isolation cannot be achieved. In order to minimize this problem and achieve correct fault isolation, a memory component $\phi_{i}{ }^{\max }(k)$ that stores the maximum value of $\phi_{i}(k)$ in a prefixed waiting 
time $T_{w}$ is used in Algorithm 1, as proposed in [41].

Additionally, to take into account the particular case where, due to the presence of uncertainty, a given fault does not trigger some of the fault indicators that are potentially affected by it according to the theoretical FSM, the strategy proposed in [42], based on considering only the components of the observed fault signature that are equal to 1 , is used.

As a result of the previous strategies, the procedure could attribute the observed fault signature to more than one fault candidate, although guaranteeing that the real fault present in the system is among the provided candidates [42]. Due to this fact, a final decision based on taking into account the correlation of the nominal residual $r^{0}(k)$ with the steady state fault sensitivities $s_{j}$ of the different fault candidates is implemented to obtain the most likely fault (see [41] for more details).

The proposed fault detection and isolation procedure is summarized in Algorithm 1. 


\section{Application results}

\subsection{Off-line design}

As it can be noticed by looking at the model structure in (1), the states are highly coupled. However, the information about the variables that are measured can be used for decoupling the system in sub-models, using structural analysis [30]. In this way, four decoupled sub-models can be obtained from equations (1)-(4), as detailed in Appendix B.

The decoupled sub-models allow obtaining the following residuals:

$$
\begin{aligned}
& r_{1} \triangleq \omega_{c p}-\hat{\omega}_{c p} \\
& r_{2} \triangleq p_{s m}-\hat{p}_{s m} \\
& r_{3} \triangleq \hat{m}_{O_{2}, 1}-\hat{m}_{O_{2}, 2} \\
& r_{4} \triangleq m_{H_{2}}-\hat{m}_{H_{2}}
\end{aligned}
$$

where the $\wedge$ indicates an estimation while the absence of $\wedge$ indicates a measurement.

It must be noticed that the mass of oxygen $m_{O_{2}}$ is not a measured variable according to the list provided in Section 2.1. Hence, a second estimation for it (the first one is provided by the cathode plus return manifold observer) is 
necessary to obtain analytical redundancy. This estimation can be computed by inverting numerically the expression that relates $m_{\mathrm{O}_{2}}$ with the measured stack voltage $v_{s t}[31]$.

The structural analysis of the system also allows determining the FSM associated to the set of faults considered in Section 2.1 and the set of residuals (39)-(42). The obtained FSM is presented in Table 2. Since all the columns in the FSM are different, all the faults are potentially isolable.

Table 2: Fault signature matrix.

\begin{tabular}{|c|c|c|c|c|c|c|}
\hline ARR & $f_{1}$ & $f_{2}$ & $f_{3}$ & $f_{4}$ & $f_{5}$ & $f_{6}$ \\
\hline$r_{1}$ & 1 & 0 & 0 & 0 & 0 & 0 \\
\hline$r_{2}$ & 0 & 1 & 0 & 0 & 1 & 0 \\
\hline$r_{3}$ & 0 & 1 & 1 & 0 & 1 & 1 \\
\hline$r_{4}$ & 0 & 1 & 1 & 1 & 0 & 0 \\
\hline
\end{tabular}

It is worth highlighting that, by looking at the FSM (Table R2-1 2) and at the equations of the decoupled sub-models (Appendix B), the importance of the availability of the measurements listed in Section 2.1 becomes clear. In fact, with the exception of the compressor speed $\omega_{c p}$ sensor (which is needed for feedback control purposes), the lack of one of the considered sensors would lead either to non-detectability/non-isolability issues (e.g. if $p_{s m}$ is not available, both the $r_{2}$ row and the $f_{2}$ column in the FSM should be 
removed, and it is straightforward to see that the faults $f_{5}$ and $f_{6}$ would not be isolable) or to the appearance of unknown inputs in the decoupled sub-models, which would impede the correct operation of the corresponding TS interval observer.

Then, a bank of TS interval observers is designed for estimating lower and upper bounds of $\omega_{c p}, p_{s m}, m_{\mathrm{O}_{2}}$ and $m_{H_{2}}$. In order to take into account the variation of the model parameters with the value of the stack current $I_{s t}$, the interval of possible values of $I_{s t} \in[100 \mathrm{~mA}, 300 \mathrm{~mA}]$ has been gridded using a step of $10 \mathrm{~mA}$, obtaining a set of 21 possible operating points and continuous-time TS representations of the ARRs. Then, in order to obtain discrete-time TS representations suitable for applying the interval observer theory introduced in Section 3, an Euler discretization with sampling time $T_{s}=0.01 s$ has been performed.

For each TS representation, a TS interval observer has been designed using the design conditions described in Section 3.3. In particular, disks of center $(0.01,0)$ and radius 0.01 have been chosen as LMI regions, in order to obtain TS interval observers whose behavior recalls the one of a predictor. Notice that in order to design the interval observer, the uncertainty must be estimated. Here, an adaptation to the TS interval observers of the approach 
proposed in [43] for interval observers is used. This approach relies on adjusting the parameter uncertainty to guarantee that no false alarms appear using data from non-faulty scenarios. Also, it has been assumed that the data coming from the sensors is affected by bounded noise with a bound of $1 \%$ of the measurement. On the other hand, a bound of $2 \%$ of the estimation has been used for the mass of oxygen $m_{\mathrm{O}_{2}}$ estimated from the stack voltage $v_{s t}$.

It is worth remarking that, from a theoretical point of view, the design conditions described in Section 3.3 guarantee the properties of the TS interval observer only at the design points. However, from a practical point of view, due to the use of a common Lyapunov matrix $P$, it is reasonable to assume that, if the gridding of the parameter space is dense enough, then the desired properties of interval bounded estimation and closed-loop pole clustering would also hold at operating points different from the design ones. A deep theoretical study of this fact is possible using the results developed by [44].

\subsection{On-line simulation results}

The proposed methodology has been applied to the case study with an extensive performance assessment phase, where simulation experiments were repeated for different faults and different magnitudes. In particular, faults 
where the value provided by the sensor corresponds to the measured value plus a percentage due to the fault are considered (e.g. $f_{1}=30 \%$ means that the compressor sensor $\omega_{c p}$ returns 1.3 times the value that would have returned in the non-faulty case).

The results of the performed simulations are shown in Tables 3-8 for each of the six available sensors. For each considered sensor fault, the rate of successful fault detection and isolation, as well as the average detection and isolation times are reported. It can be seen that, for all the sensors, there are critical fault magnitudes: for magnitudes above the upper critical one (e.g. $f_{1}=6 \%$ for the compressor sensor $\omega_{c p}$ ), the performances of the fault diagnosis approach are very good, since correct fault detection and isolation are always achieved within a small time. On the other hand, for fault magnitudes below the lower critical one (e.g. $f_{1}=5 \%$ for $\omega_{c p}$ ), fault detection is not achievable, i.e. those faults are not detectable. Between these two extremes, the behavior of the fault diagnosis approach varies depending on the considered fault. In some cases, the smaller the fault magnitude is, the smaller the rate of successful fault detection and isolation is, but with the detection/isolation time remaining unaffected. This is the case of the faults in $\omega_{c p}$ and $m_{H_{2}}$. In the remaining cases, the effect of decreasing the 
Table 3: Results of fault diagnosis for $f_{1}\left(\omega_{c p}\right.$ sensor fault).

\begin{tabular}{|c|c|c|c|c|}
\hline$f_{1}$ & $\begin{array}{c}\text { Successful } \\
\text { fault detection }\end{array}$ & $\begin{array}{c}\text { Successful } \\
\text { fault isolation }\end{array}$ & $\bar{t}_{d}$ & $\bar{t}_{i}$ \\
\hline$\geq 6.6 \%$ & $100 \%$ & $100 \%$ & $0.01 s$ & $0.01 s$ \\
\hline $6.2 \%, 6.4 \%$ & $95 \%$ & $95 \%$ & $0.01 s$ & $0.01 s$ \\
\hline $5.8 \%, 6 \%$ & $80 \%$ & $80 \%$ & $0.01 s$ & $0.01 s$ \\
\hline $5.6 \%$ & $55 \%$ & $55 \%$ & $0.01 s$ & $0.01 s$ \\
\hline $5.4 \%$ & $35 \%$ & $35 \%$ & $0.01 s$ & $0.01 s$ \\
\hline $5.2 \%$ & $15 \%$ & $15 \%$ & $0.01 s$ & $0.01 s$ \\
\hline$\leq 5 \%$ & $0 \%$ & $0 \%$ & - & - \\
\hline
\end{tabular}

Table 4: Results of fault diagnosis for $f_{2}\left(p_{s m}\right.$ sensor fault).

\begin{tabular}{|c|c|c|c|c|}
\hline$f_{2}$ & $\begin{array}{c}\text { Successful } \\
\text { fault detection }\end{array}$ & $\begin{array}{c}\text { Successful } \\
\text { fault isolation }\end{array}$ & $\bar{t}_{d}$ & $\bar{t}_{i}$ \\
\hline$\geq 6 \%$ & $100 \%$ & $100 \%$ & $0.01 s$ & $0.01 s$ \\
\hline $5.5 \%$ & $100 \%$ & $100 \%$ & $0.015 s$ & $0.015 s$ \\
\hline$[3.5 \%, 5 \%]$ & $100 \%$ & $100 \%$ & $0.02 s$ & $0.03 s$ \\
\hline $3 \%$ & $100 \%$ & $100 \%$ & $0.02 s$ & $0.031 s$ \\
\hline $2.5 \%$ & $100 \%$ & $100 \%$ & $0.022 s$ & $0.034 s$ \\
\hline $2 \%$ & $100 \%$ & $100 \%$ & $0.033 s$ & $0.055 s$ \\
\hline $1.5 \%$ & $100 \%$ & $80 \%$ & $0.103 s$ & $0.694 s$ \\
\hline $1 \%$ & $10 \%$ & $0 \%$ & $0.850 s$ & - \\
\hline$\leq 0.5 \%$ & $0 \%$ & $0 \%$ & - & - \\
\hline
\end{tabular}

fault magnitude is visible on the detection/isolation time.

Moreover, to better illustrate the proposed method, let us consider the following two fault scenarios:

- Fault scenario 1: $f_{2}=30 \%$ (fault in the supply manifold sensor $p_{\text {sm }}$ ), starting from $t=15 \mathrm{~s}$

- Fault scenario 2: $f_{4}=10 \%$ (fault in the anode pressure sensor $p_{a n}$ ), 
Table 5: Results of fault diagnosis for $f_{3}$ ( $v_{s t}$ sensor fault).

\begin{tabular}{|c|c|c|c|c|}
\hline$f_{3}$ & $\begin{array}{c}\text { Successful } \\
\text { fault detection }\end{array}$ & $\begin{array}{c}\text { Successful } \\
\text { fault isolation }\end{array}$ & $\bar{t}_{d}$ & $\bar{t}_{i}$ \\
\hline$\geq 3.5 \%$ & $100 \%$ & $100 \%$ & $0.01 s$ & $0.01 s$ \\
\hline $3 \%$ & $100 \%$ & $100 \%$ & $2.361 s$ & $2.361 s$ \\
\hline $2.5 \%$ & $100 \%$ & $100 \%$ & $9.597 s$ & $9.597 s$ \\
\hline $2 \%$ & $100 \%$ & $100 \%$ & $11.507 s$ & $11.507 s$ \\
\hline $1.5 \%$ & $100 \%$ & $100 \%$ & $11.998 s$ & $11.998 s$ \\
\hline$\leq 1 \%$ & $0 \%$ & $0 \%$ & - & - \\
\hline
\end{tabular}

Table 6: Results of fault diagnosis for $f_{4}\left(m_{H 2}\right.$ sensor fault).

\begin{tabular}{|c|c|c|c|c|}
\hline$f_{4}$ & $\begin{array}{c}\text { Successful } \\
\text { fault detection }\end{array}$ & $\begin{array}{c}\text { Successful } \\
\text { fault isolation }\end{array}$ & $\bar{t}_{d}$ & $\bar{t}_{i}$ \\
\hline$\geq 14 \%$ & $100 \%$ & $100 \%$ & $0.01 s$ & $0.02 s$ \\
\hline $13 \%$ & $95 \%$ & $95 \%$ & $0.01 s$ & $0.02 s$ \\
\hline $12 \%$ & $85 \%$ & $85 \%$ & $0.01 s$ & $0.02 s$ \\
\hline $11 \%$ & $70 \%$ & $70 \%$ & $0.01 s$ & $0.02 s$ \\
\hline $10 \%$ & $60 \%$ & $60 \%$ & $0.01 s$ & $0.02 s$ \\
\hline $9 \%$ & $20 \%$ & $20 \%$ & $0.01 s$ & $0.02 s$ \\
\hline $8 \%$ & $15 \%$ & $15 \%$ & $0.01 s$ & $0.02 s$ \\
\hline$\leq 7 \%$ & $0 \%$ & $0 \%$ & $0.01 s$ & - \\
\hline
\end{tabular}

Table 7: Results of fault diagnosis for $f_{5}$ ( $p_{c a}$ sensor fault).

\begin{tabular}{|c|c|c|c|c|}
\hline$f_{5}$ & $\begin{array}{c}\text { Successful } \\
\text { fault detection }\end{array}$ & $\begin{array}{c}\text { Successful } \\
\text { fault isolation }\end{array}$ & $\bar{t}_{d}$ & $\bar{t}_{i}$ \\
\hline$\geq 25 \%$ & $100 \%$ & $100 \%$ & $0.02 s$ & $0.02 s$ \\
\hline $20 \%$ & $100 \%$ & $100 \%$ & $0.02 s$ & $0.026 s$ \\
\hline $15 \%$ & $100 \%$ & $100 \%$ & $0.021 s$ & $0.078 s$ \\
\hline $10 \%$ & $10 \%$ & $0 \%$ & $0.03 s$ & - \\
\hline $5 \%$ & $0 \%$ & $0 \%$ & - & - \\
\hline
\end{tabular}


Table 8: Results of fault diagnosis for $f_{6}$ ( $p_{a n}$ sensor fault).

\begin{tabular}{|c|c|c|c|c|}
\hline$f_{6}$ & $\begin{array}{c}\text { Successful } \\
\text { fault detection }\end{array}$ & $\begin{array}{c}\text { Successful } \\
\text { fault isolation }\end{array}$ & $\bar{t}_{d}$ & $\bar{t}_{i}$ \\
\hline$\geq 3 \%$ & $100 \%$ & $100 \%$ & $0.02 s$ & $0.02 s$ \\
\hline $2.5 \%$ & $100 \%$ & $100 \%$ & $0.022 s$ & $0.025 s$ \\
\hline $2 \%$ & $100 \%$ & $100 \%$ & $0.031 s$ & $0.034 s$ \\
\hline $1.5 \%$ & $100 \%$ & $100 \%$ & $0.218 s$ & $0.231 s$ \\
\hline$\leq 1 \%$ & $0 \%$ & $0 \%$ & - & - \\
\hline
\end{tabular}

starting from $t=15 \mathrm{~s}$

Figs. 2-4 show the measured value (red line), lower interval estimation (blue line) and upper interval estimation (black line) in the first fault scenario, for the residuals corresponding to $p_{s m}, m_{\mathrm{O}_{2}}$ and $m_{H_{2}}$ that are sensitive to this fault according to the FSM presented in Table 2. Notice that while the fault is not present, the measurement is inside the interval estimation provided by the corresponding TS interval observer. However, when the fault appears (at $t=15 \mathrm{~s}$ ), the measured values are outside the intervals estimated by the TS interval observers, leading to the observer fault signature given by $\phi_{2}, \phi_{3}$ and $\phi_{4}$ shown in Fig. 5. By matching this observed fault signature with the FSM presented in Table 2, the fault in the supply manifold sensor $p_{s m}$ can be diagnosed.

On the other hand, in the second fault scenario, the fault affects only the residual corresponding to $m_{H_{2}}$ (see Fig. 6) according to the FSM matrix 


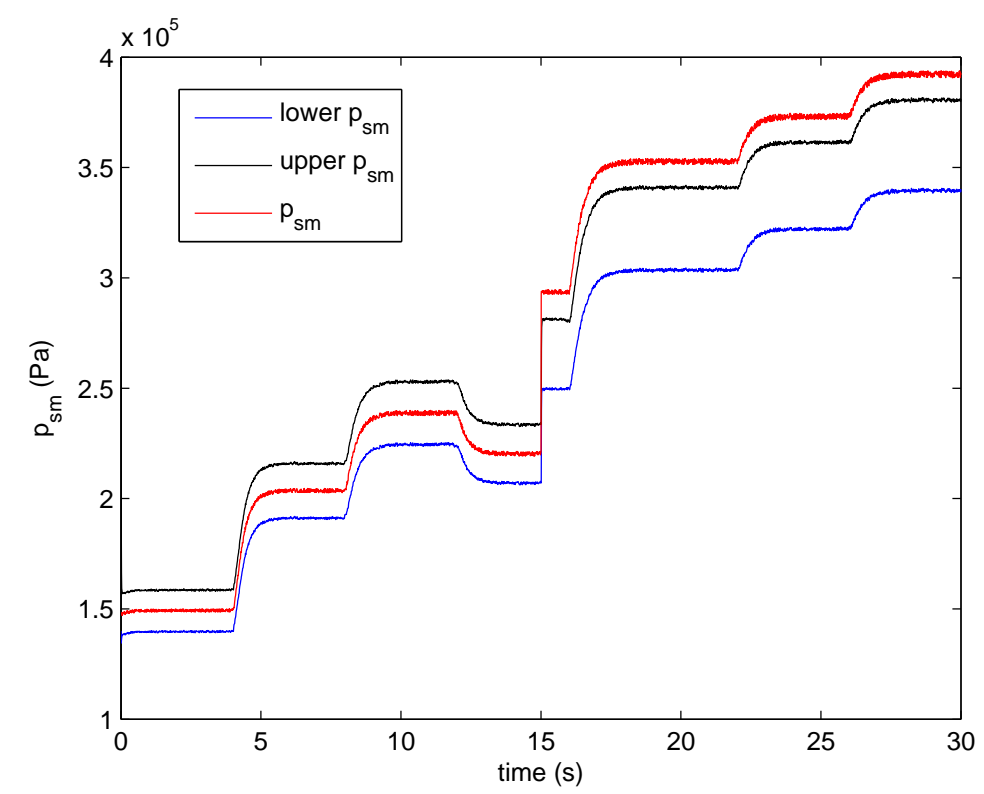

Figure 2: $p_{s m}, \underline{p}_{s m}$ and $\bar{p}_{s m}$ with $f_{2}=30 \%$ (fault in the supply manifold sensor $p_{s m}$ ).

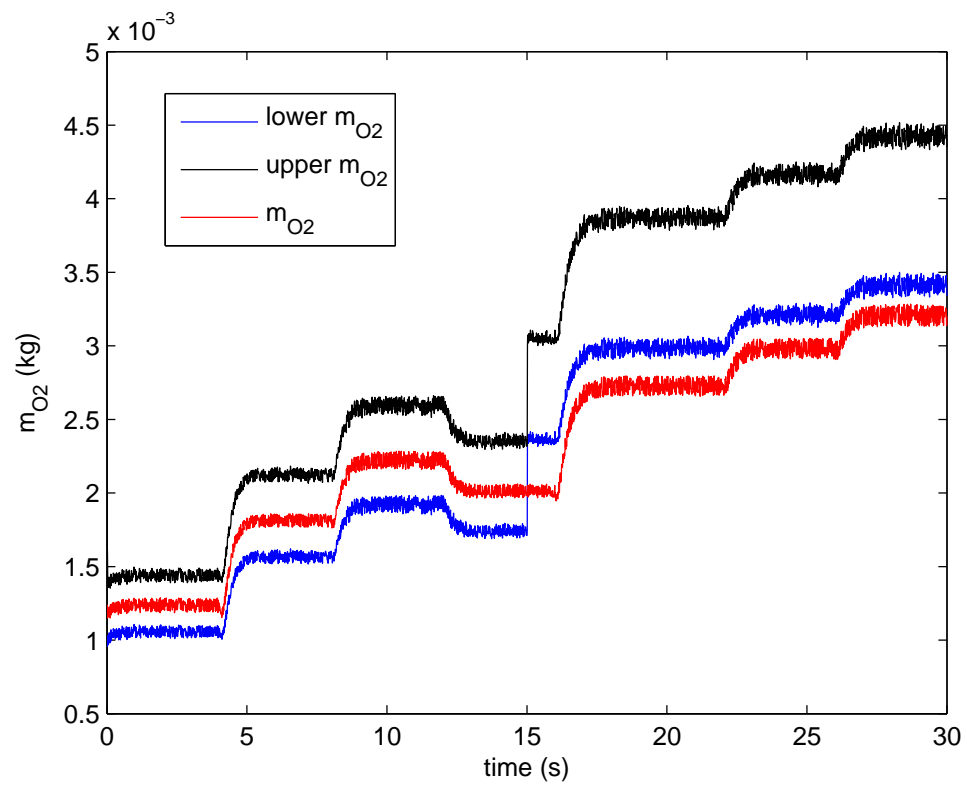

Figure 3: $m_{O 2}, \underline{m}_{O 2}$ and $\bar{m}_{O 2}$ with $f_{2}=30 \%$ (fault in the supply manifold sensor $p_{s m}$ ). 


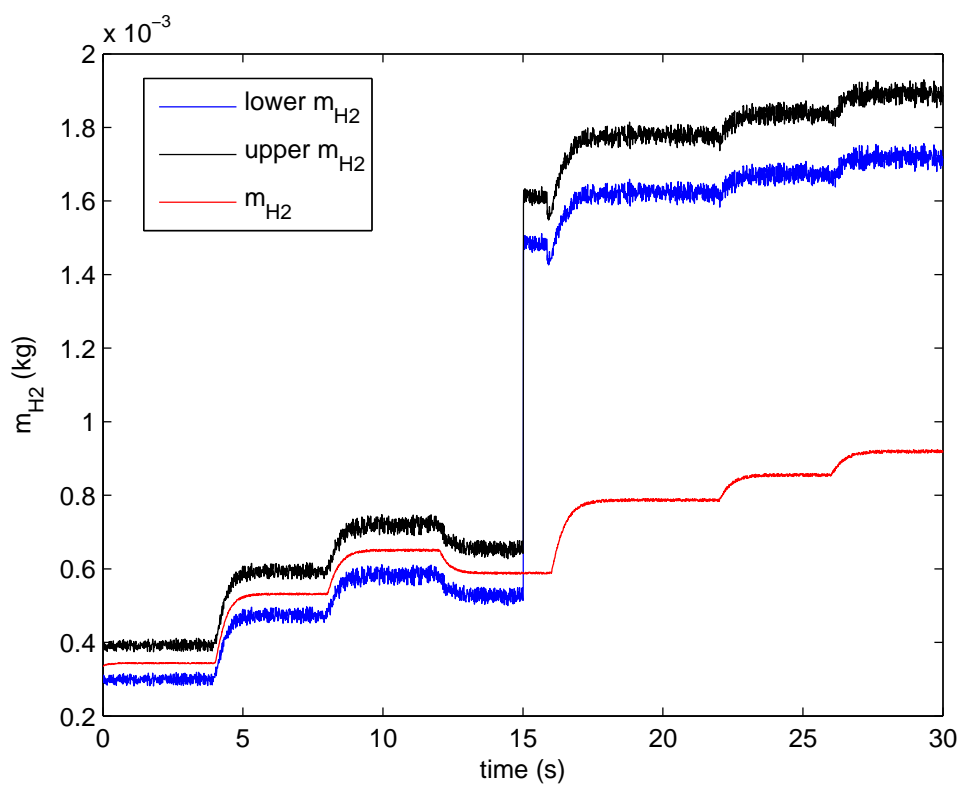

Figure 4: $m_{H 2}, \underline{m}_{H 2}$ and $\bar{m}_{H 2}$ with $f_{2}=30 \%$ (fault in the supply manifold sensor $p_{s m}$ ).
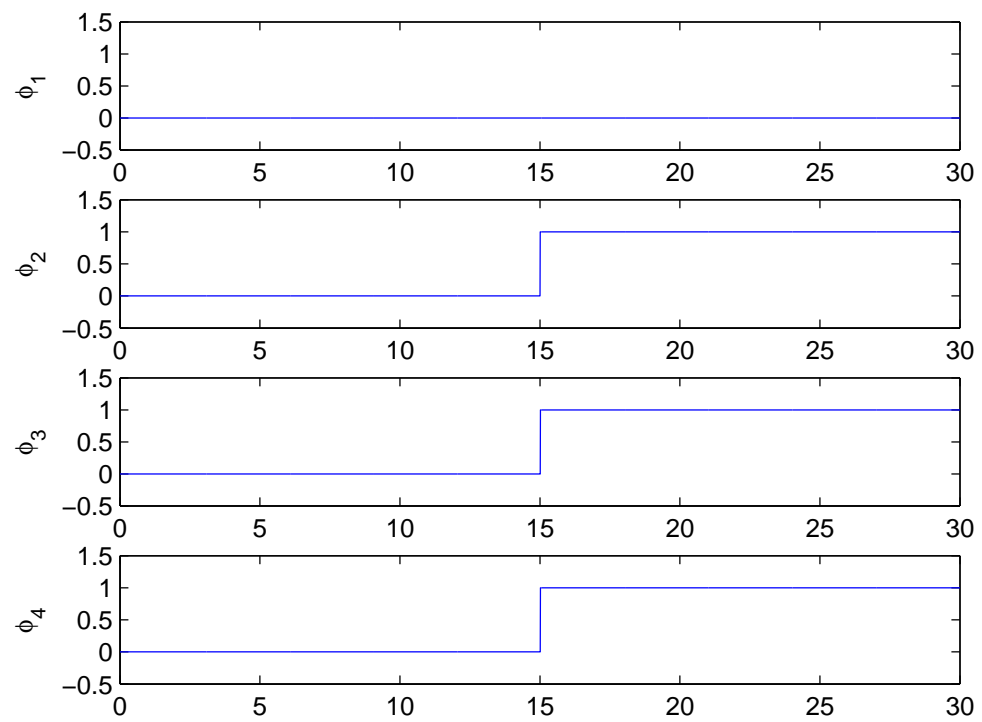

Figure 5: Fault signatures with $f_{2}=30 \%$ (fault in the supply manifold sensor $p_{s m}$ ). 
presented in Table 2. As in the case of the first fault scenario, before the fault appearance, the measurement $m_{H_{2}}$ is inside the interval estimation provided by the corresponding TS interval observer, as shown in Fig. 7. However, after the fault occurs, the measurement goes out of the estimation interval, allowing to detect the fault generating the observed fault signature $\phi_{4}$ that, according to Table 2, allows diagnosing the fault in the anode pressure sensor $p_{a n}$.

In order to conclude the performance analysis, the proposed approach has been compared to a fault diagnoser based on fixed thresholds. In fact, the main advantage of the proposed robust fault diagnosis using a TS interval observer approach is that it is able to generate adaptive thresholds that guarantee that no false alarms occur due to the model uncertainty. As shown in Table 9, where the minimum fault magnitudes that assure a $100 \%$ successful fault isolation rate are listed, the conservatism provided by fixed thresholds leads to worse performances than the proposed approach.

\section{Conclusions}

In this paper, the problem of robust fault diagnosis of PEM fuel cells has been addressed by using a bank of TS interval observers that consider 
Table 9: Minimum fault magnitudes that assure a 100\% successful fault isolation rate.

\begin{tabular}{|c|c|c|}
\hline & Adaptive thresholds & Fixed thresholds \\
\hline Fault $1 \omega_{c p}$ & $6.5 \%$ & $9.2 \%$ \\
\hline Fault $2 p_{s m}$ & $1.8 \%$ & $3.4 \%$ \\
\hline Fault $3 v_{s t}$ & $1.4 \%$ & $1.4 \%$ \\
\hline Fault $4 m_{H_{2}}$ & $13.4 \%$ & $22 \%$ \\
\hline Fault $5 p_{c a}$ & $11.4 \%$ & $22 \%$ \\
\hline Fault $6 p_{a n}$ & $1.4 \%$ & $3.3 \%$ \\
\hline
\end{tabular}

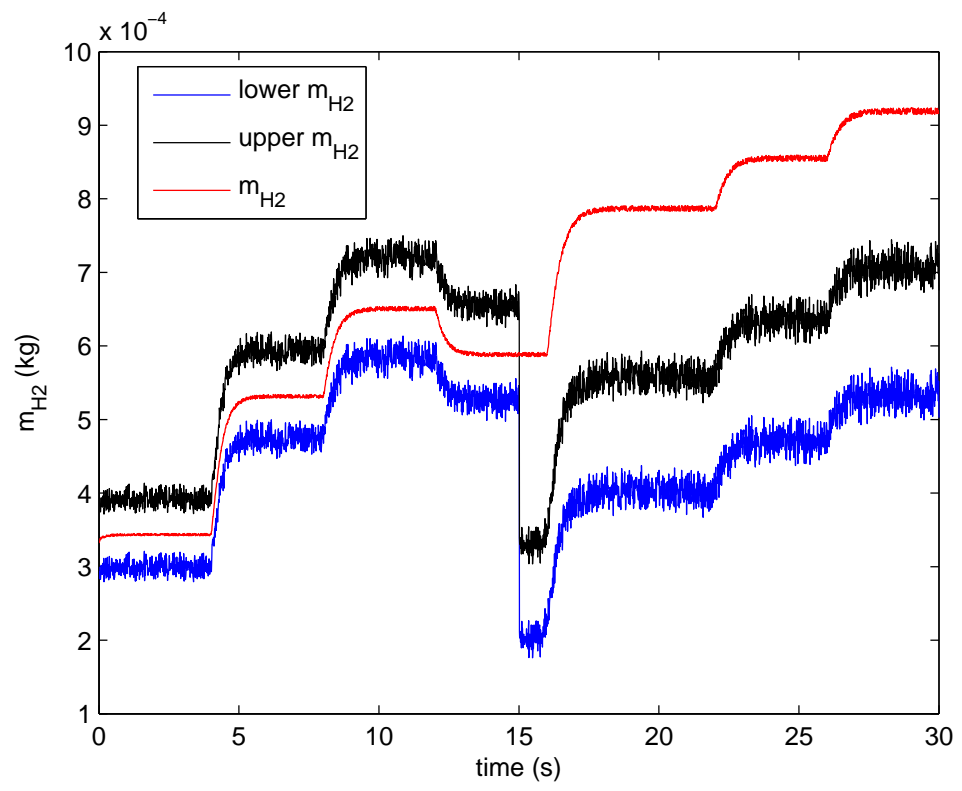

Figure 6: $m_{H 2}, \underline{m}_{H 2}$ and $\bar{m}_{H 2}$ with $f_{6}=10 \%$ (fault in the anode pressure sensor $p_{a n}$ ). 

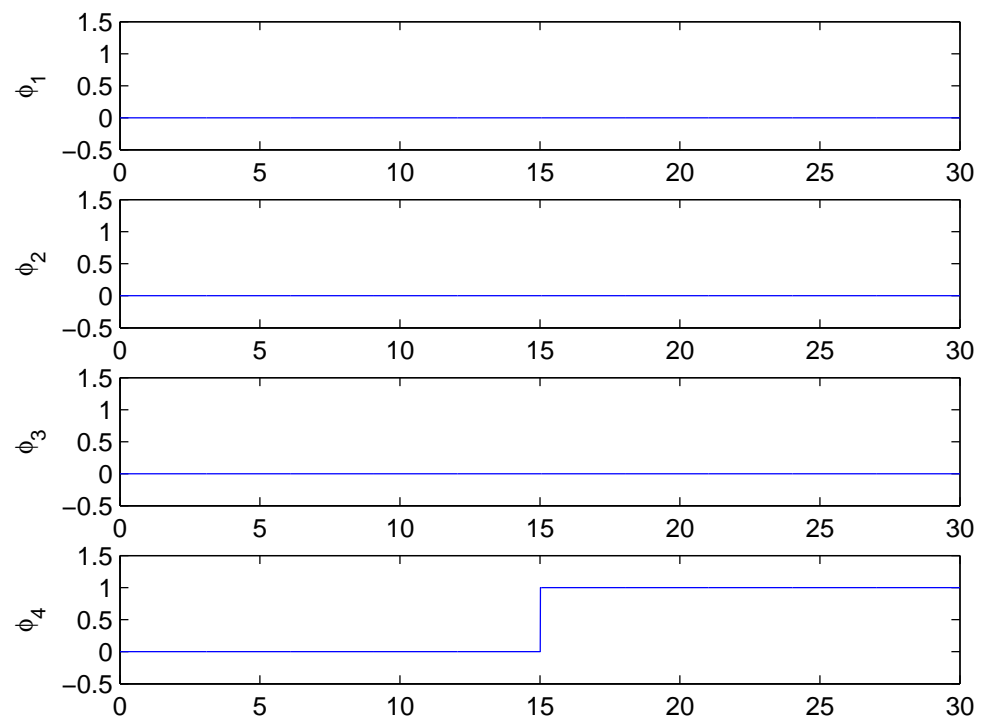

Figure 7: Fault signatures with $f_{6}=10 \%$ (fault in the anode pressure sensor $p_{a n}$ ).

uncertainty in a bounded context. The design of the TS interval observers is performed using LMIs and calibrating the uncertainty in such a way that, in a non-faulty situation, the measurements will be inside the computed intervals, thus avoiding the possibility of false alarms. Fault detection is based on checking whether all the measurements lie inside their corresponding estimated interval bounds. In the case a fault is detected, the measurements that are inconsistent with their corresponding estimations are annotated and a fault isolation procedure is triggered. By using the theoretical fault signature matrix, the fault is isolated by means of a logic reasoning that takes into account the presence of bounded uncertainty and, if the number of candidate 
faults is more than one, a posterior correlation analysis provides the most probable fault.

The proposed approach has been successfully tested using a well known PEM fuel case study proposed in the literature. It has been shown that as long as the fault magnitudes are above some critical value, correct fault detection and isolation is achieved. On the other hand, for each considered fault, there is a lower critical value, below which fault detection cannot be achieved, i.e. the fault is not detectable. Between these two extreme cases, the performance of the fault diagnoser increases when the fault magnitude increases.

The obtained results are satisfactory and the proposed strategy is promising. Future research includes its application to a real set-up, as well as to extend the proposed approach to other types of faults, e.g. affecting the actuators, and integrate the proposed fault diagnosis methodology with some fault tolerant control strategy in order to increase the reliability and performance of PEM fuel cells under fault occurrence. 


\section{Appendix A. Proof of Theorem 1}

If (32) holds, the dynamics of the interval estimation errors $\underline{e}(k)=x(k)-$ $\underline{x}(k)$ and $\bar{e}(k)=\bar{x}(k)-x(k)$ are given by:

$$
\begin{aligned}
& \underline{e}(k+1)=\left(A_{0 i}-\underline{L}_{i} C\right) \underline{e}(k)+\sum_{j=1}^{3} \underline{w}_{i}^{j}(k) \\
& \bar{e}(k+1)=\left(A_{0 i}-\bar{L}_{i} C\right) \bar{e}(k)+\sum_{j=1}^{3} \bar{w}_{i}^{j}(k) \\
& \underline{w}_{i}^{1}(k)=\Delta A_{i} x(k)-\underline{\Delta A_{i}}{ }^{+} \underline{x}^{+}(k)+\overline{\Delta A_{i}} \underline{x}^{+}(k) \\
& +\underline{\Delta A_{i}}{ }^{-} \bar{x}^{+}(k)-{\overline{\Delta A_{i}}}^{-} \bar{x}^{-}(k) \\
& \underline{w}_{i}^{2}(k)=\left|\underline{L_{i}}\right| V(k) E_{n_{y}}-\underline{L_{i}} v(k) \\
& \underline{w}_{i}^{3}(k)=d(k)-\underline{d}(k) \\
& \bar{w}_{i}^{1}(k)=-\Delta A_{i} x(k)+{\overline{\Delta A_{i}}}^{+} \bar{x}^{+}(k)-\underline{\Delta A_{i}}{ }^{+} \bar{x}^{-}(k) \\
& -{\overline{\Delta A_{i}}}^{-} \underline{x}^{+}(k)+\underline{\Delta A_{i}}{ }^{-} \underline{x}^{-}(k) \\
& \bar{w}_{i}^{2}(k)=\left|\overline{L_{i}}\right| V(k) E_{n_{y}}+\overline{L_{i}} v(k) \\
& \bar{w}_{i}^{3}(k)=\bar{d}(k)-d(k)
\end{aligned}
$$


Following [25], if (24) holds, then:

$$
A_{0 i}-\underline{L_{i}} C, A_{0 i}-\overline{L_{i}} \in \mathbb{R}_{+}^{n \times n}
$$

where $\mathbb{R}_{+}^{n \times n}$ denotes the set of real matrices with nonnegative elements. Hence, the dynamics for $\underline{e}(k)$ and $\bar{e}(k)$ is cooperative [45] and (13) holds as long as $\underline{w}_{i}^{j}(k) \geq 0$ and $\bar{w}_{i}^{j}(k) \geq 0 \forall i=1, \ldots, N, \forall j=1, \ldots, 3$ and $\forall k \geq 0$, which is true due to the assumptions (10)-(12) [25].

In order to show that $\underline{x}(k)$ and $\bar{x}(k)$ remain bounded for all $t \geq 0$, the equations in (14) are rewritten as:

$$
\begin{aligned}
& \underline{x_{i}}(k+1)=\left[A_{0 i}-\underline{L_{i}} C+\underline{\Delta A_{i}}{ }^{+}\right] \underline{x}(k)+\underline{f_{i}}(\underline{x}(k), \bar{x}(k))+\underline{\delta_{i}}(k) \\
& \overline{x_{i}}(k+1)=\left[A_{0 i}-\overline{L_{i}} C+\overline{\Delta A_{i}}\right] \bar{x}(k)+\overline{f_{i}}(\underline{x}(k), \bar{x}(k))+\overline{\delta_{i}}(k)
\end{aligned}
$$

with:

$$
\begin{aligned}
\underline{f_{i}}(\underline{x}(k), \bar{x}(k))= & \left(\underline{\Delta A_{i}}{ }^{+}-{\overline{\Delta A_{i}}}^{+}\right) \underline{x}^{-}(k) \\
& -\underline{\Delta A_{i}} \bar{x}^{+}(k)+\overline{\Delta A_{i}} \bar{x}^{-}(k) \\
\overline{f_{i}}(\underline{x}(k), \bar{x}(k))= & \left(\overline{\Delta A_{i}}{ }^{+}-\underline{\Delta A_{i}}{ }^{+}\right) \bar{x}^{-}(k) \\
& -{\overline{\Delta A_{i}}}^{-} \underline{x}^{+}(k)+\underline{\Delta A_{i}}{ }^{-} \underline{x}^{-}(k)
\end{aligned}
$$




$$
\begin{aligned}
& \underline{\delta_{i}}(k)=\underline{L_{i}} y(k)-\left|\underline{L_{i}}\right| V(k) E_{n_{y}}+\underline{d}(k) \\
& \overline{\delta_{i}}(k)=\overline{L_{i}} y(k)-\left|\overline{L_{i}}\right| V(k) E_{n_{y}}+\bar{d}(k)
\end{aligned}
$$

Then, the boundedness of $\underline{x}(k)$ and $\bar{x}(k)$ is a consequence of the nonnegativity of $A_{0 i}-\underline{L}_{i} C+\underline{\Delta A_{i}}{ }^{+}$and $A_{0 i}-\bar{L}_{i} C+{\overline{\Delta A_{i}}}^{+}$, the boundedness of the inputs $\underline{\delta_{i}}(k)$ and $\overline{\delta_{i}}(k)$ and the property of the functions $\underline{f_{i}}$ and $\overline{f_{i}}$ of being globally Lipschitz [25], and is proved by introducing the system:

$$
\xi(k+1)=G_{i} \xi(k)+\phi_{i}(\xi(k))+\delta_{i}(k)
$$

where:

$$
\begin{gathered}
\xi(k)=\left(\begin{array}{c}
\underline{x}(k) \\
\bar{x}(k)
\end{array}\right) \phi_{i}(\xi(k))=\left(\begin{array}{c}
\underline{f}_{i}(\xi(k)) \\
\bar{f}_{i}(\xi(k))
\end{array}\right) \delta_{i}(k)=\left(\begin{array}{c}
\underline{\delta}_{i}(k) \\
\bar{\delta}_{i}(k)
\end{array}\right) \\
G_{i}=D_{i}-\left(\begin{array}{cc}
\underline{L}_{i} & 0 \\
0 & \bar{L}_{i}
\end{array}\right) \Upsilon
\end{gathered}
$$

and:

$$
\left|\phi_{i}(\xi(k))\right| \leq \eta|\xi(k)|
$$

with $\eta$ defined as in (28). In fact, using Schur complement [46], it can be 
shown that if (23) holds, the following is true for the increment $\Delta \mathcal{V}(k)$ of the Lyapunov function $\mathcal{V}(k)=\xi(k)^{T} P \xi(k)[25]$ :

$$
\Delta \mathcal{V}(k) \leq-\xi(k)^{T} Q \xi(k)+\left(1+\varepsilon_{1}^{-1}+\varepsilon_{2}^{-1}\right) \delta_{i}(k)^{T} P \delta_{i}(k)
$$

that proves the boundedness of $\underline{x}(k)$ and $\bar{x}(k)[47]$.

Finally, it must be shown that the closed-loop poles of the TS interval observer are in $\mathcal{D}$, defined as in (18). This is straightforward, by taking into account that the closed-loop matrix of the TS interval observer is given by:

$$
A_{c l, i}=\left(\begin{array}{cc}
A_{0, i} & 0 \\
0 & A_{0, i}
\end{array}\right)-\left(\begin{array}{cc}
\underline{L}_{i} & 0 \\
0 & \bar{L}_{i}
\end{array}\right) \Upsilon
$$

such that (25) is obtained from the direct application of Theorem 2.2 in [34] to $A_{c l, i}^{T}$. This completes the proof.

\section{Appendix B. Decoupled sub-models}

For illustrative purpose, the first sub-model obtained by applying structural analysis [30] to the linear-like model of the PEMFC (1) is completely detailed in the following. For the remaining three sub-models, only their structure is provided. 


\section{Compressor}

Starting from the non-linear equation for the compressor provided in [14], and by approximating intermediate variables and terms as functions of the stack current $I_{s t}$, the following sub-model for the compressor can be obtained:

$$
\dot{\omega}_{c p}=\tilde{a}_{11} \omega_{c p}+\tilde{c} t_{1}
$$

with:

$$
\begin{aligned}
\tilde{a}_{11} & =-\frac{\tilde{z}_{3} v_{c p}}{J_{c p} R_{c m}} k_{v} \\
\tilde{c} t_{1} & =\frac{\tilde{z}_{3}}{J_{c p}}\left(\frac{v_{c p}^{2}}{R_{c m}}-\frac{C_{p} T_{a m b}}{\eta_{c m} \tilde{\eta}_{c p}} \tilde{z}_{1} \tilde{z}_{2}\right) \\
v_{c p} & =0.672519 I_{s t}+33.554115 \\
\tilde{\eta}_{c p} & \approx 0.777217 \\
\tilde{z}_{1} & \approx 0.275641 \cdot 10^{-3} I_{s t}-0.340993 \cdot 10^{-3} \\
\tilde{z}_{2} & \approx 0.001375 I_{s t}-0.023710 \\
\tilde{z}_{3} & \approx-0.000426 \cdot 10^{-3} I_{s t}+0.213459 \cdot 10^{-3}
\end{aligned}
$$

where $k_{v}=0.0153 \mathrm{~V} /(\mathrm{rad} / \mathrm{s})$ is the motor electric constant, $J_{c p}=5 \times 10^{-5} \mathrm{~kg}$. $m^{2}$ is the compressor and motor inertia, $R_{c m}=0.816 \Omega$ is the compressor 
motor circuit resistance, $C_{p}=1004 \mathrm{~J} /(\mathrm{kg} \cdot \mathrm{K})$ is the air heat capacity at constant pressure, $T_{a m b}=298 K$ is the ambient temperature and $\eta_{c m}=0.9$ is the compressor efficiency.

\section{Supply manifold}

The supply manifold sub-model is given by the following expression:

$$
\dot{p}_{s m}=\tilde{a}_{22} p_{s m}+\tilde{b}_{25} p_{c a}+\tilde{c} t_{2}
$$

\section{Cathode plus return manifold}

The cathode plus manifold sub-model is a second order state space model, where the pressure at the return manifold $p_{r m}$ is a state variable that is not measured. It can be expressed as:

$$
\begin{aligned}
{\left[\begin{array}{c}
\dot{m}_{O_{2}} \\
\dot{p}_{r m}
\end{array}\right]=} & {\left[\begin{array}{cc}
\tilde{a}_{77} & \tilde{a}_{78} \\
0 & \tilde{a}_{88}
\end{array}\right]\left[\begin{array}{l}
m_{O_{2}} \\
p_{r m}
\end{array}\right]+\left[\begin{array}{c}
\tilde{b}_{75} \\
b_{85}
\end{array}\right] p_{c a} } \\
& +\left[\begin{array}{c}
b_{72} \\
0
\end{array}\right] p_{s m}+\left[\begin{array}{c}
b_{70} \\
0
\end{array}\right] I_{s t}+\left[\begin{array}{c}
\tilde{c} t_{7} \\
0
\end{array}\right]
\end{aligned}
$$




\section{Anode}

Finally, the anode sub-model is expressed as follows:

$$
\dot{m}_{H_{2}}=\tilde{b}_{32} p_{s m}+\tilde{b}_{34} p_{a n}+b_{30} I_{s t}+\tilde{c} t_{3}
$$

\section{Acknowledgements}

This work has been funded by the Spanish MINECO through the projects CICYT ECOCIS (ref. DPI2013-48243-C2-1-R) and CICYT HARCRICS (ref. DPI2014-58104-R), by AGAUR through the contracts FI-DGR 2014 (ref. 2014FI_B1 00172) and FI-DGR 2015 (ref. 2015FI_B2 00171) and by the

DGR of Generalitat de Catalunya (SAC group Ref. 2014/SGR/374).

\section{References}

[1] A. Yilanci, I. Dincer, H. Z. Ozturk, Performance analysis of a PEM fuel cell unit in a solar-hydrogen system, International Journal of Hydrogen Energy 33 (2008) 7538-7552.

[2] J. T. Pukrushpan, A. G. Stefanopoulou, H. Peng, Control of Fuel Cell Power Systems: Principles, Modeling, Analysis, and Feedback Design, Springer-Verlag, London, 2004. 
[3] A. Niknezhadi, M. Allué-Fantova, C. Kunusch, C. Ocampo-Martínez, Design and implementation of LQR/LQG strategies for oxygen stoichiometry control in PEM fuel cells based systems, Journal of Power Sources 196 (2011) 4277-4282.

[4] C. Ziogou, S. Papadopoulou, M. C. Georgiadis, S. Voutetakis, On-line nonlinear model predictive control of a PEM fuel cell system, Journal of Process Control 23 (2013) 483 - 492.

[5] I. Matraji, S. Laghrouche, M. Wack, Pressure control in a PEM fuel cell via second order sliding mode, International Journal of Hydrogen Energy 37 (2012) 16104-16116.

[6] J. Wu, X. Yuan, J. Martin, Z. J. Wang, H., J. Shen, S. Wu, W. Merida, A review of PEM fuel cell durability: degradation mechanisms and mitigation strategies, Journal of Power Sources 184 (2008) 104-119.

[7] W. Schmittinger, A. Vahidi, A review of the main parameters influencing long-term performance and durability of PEM fuel cells, Journal of Power Sources 180 (2008) 1-14.

[8] S. Knights, K. Colbow, J. St-Pierre, D. Wilkinson, Aging mechanisms 
and lifetime of PEFC and DMFC, Journal of Power Sources 127 (2004) $127-134$.

[9] N. Yousfi-Steiner, P. H. Moçotéguy, D. Candusso, D. Hissel, A. Hernandez, A. Aslanides, A review on PEM voltage degradation associated with water management: impacts, influent factors and characterization, Journal of Power Sources 183 (2008) 260-274.

[10] N. Yousfi-Steiner, P. Moçotéguy, D. Candusso, D. Hissel, A review on polymer electrolyte membrane fuel cell catalyst degradation and starvation issues: causes, consequences and diagnostic for mitigation, Journal of Power Sources 194 (2009) 130-145.

[11] S. A. Gregoriev, K. A. Dzhus, D. G. Bessarabov, P. Millet, Failure of PEM water electrolysis cells: case study involving anode dissolution and membrane thinning, International Journal of Hydrogen Energy 39 (2014) 20440-20446.

[12] R. Petrone, Z. Zheng, D. Hissel, M. Péra, C. Pianese, M. Sorrentino, M. Becherif, N. Yousfi-Steiner, A review on model-based diagnosis methodologies for PEMFCs, International Journal of Hydrogen Energy 38 (2013) 7077-7091. 
[13] A. M. Dhirde, N. V. Dale, H. Salehfar, M. D. Mann, T. H. Han, Equivalent electric circuit modeling and performance analysis of a PEM fuel cell stack using impedance spectroscopy, IEEE Transactions on Energy Conversion 25 (2010) 778-786.

[14] J. T. Pukrushpan, H. Peng, A. G. Stefanopoulou, Control-oriented modeling and analysis for automotive fuel cell systems, Journal of Dynamics Systems, Measurement, and Control 126 (2004) 14-25.

[15] S. V. Puranik, A. Keyhani, F. Khorrami, State-space modeling of proton exchange membrane fuel cell, IEEE Transactions on Energy Conversion 25 (2010) 804-813.

[16] R. Saisset, G. Fontes, C. Turpin, S. Astier, Bond Graph model of a PEM fuel cell, Journal of Power Sources 156 (2006) 100-107.

[17] F. D. Bianchi, C. Kunusch, , C. Ocampo-Martínez, R. S. Sánchez-Peña, A gain-scheduled LPV control for oxygen stoichiometry regulation in PEM fuel cell systems, IEEE Transactions on Control Systems Techonology $22(2014)$ 1837-1844.

[18] S. de Lira, V. Puig, J. Quevedo, A. Husar, LPV observer design for 
PEM fuel cell system: Application to fault detection, Journal of Power Sources 196 (2011) $4298-4305$.

[19] T. Takagi, M. Sugeno, Fuzzy identification of systems and its applications to modeling and control, IEEE Transactions on Systems, Man, and Cybernetics SMC-15 (1985) 116-132.

[20] S. Olteanu, A. Aitouche, M. Oueidat, A. Jouni, PEM fuel cell modeling and simulation via the Takagi-Sugeno Fuzzy model, in: International Conference on Renewable Energies for Developing Countries (REDEC), pp. 1-7.

[21] D. Ichalal and B. Marx and J. Ragot and D. Maquin, Fault detection, isolation and estimation for Takagi-Sugeno nonlinear systems, Journal of The Franklin Institute 351 (2014) 36513676.

[22] S. Delrot and T.M. Guerra and M. Dambrine and F. Delmotte, Fouling detection in a heat exchanger by observer of Takagi-Sugeno type for systems with unknown polynomial inputs, Engineering Applications of Artificial Intelligence 25 (2012) 15581566.

[23] Z. Gao, X. Shi, S. Ding, Fuzzy state/disturbance observer design for T/S fuzzy systems with application to sensor fault estimation, Systems, 
Man, and Cybernetics, Part B: Cybernetics, IEEE Transactions on 38 (2008) 875-880.

[24] E. Kamal, A. Aitouche, R. Ghorbani, M. Bayart, Robust fuzzy faulttolerant control of wind energy conversion systems subject to sensor faults, IEEE Transactions on Sustainable Energy 3 (2013) 231-241.

[25] D. Efimov, T. Raïssi, W. Perruquetti, A. Zolghadi, Estimation and control of discrete-time LPV systems using interval observers, in: Proceedings of the IEEE 52nd Annual Conference on Decision and Control (CDC), pp. 5036-5041.

[26] B. Olivier, J. Gouzé, Closed loop observers bundle for uncertain biotechnological models, Journal of Process Control 14 (2004) 765-774.

[27] M. Moisan, O. Bernard, J. Gouzé, Near optimal interval observers bundle for uncertain bio-reactors, Automatica 45 (2009) 291-295.

[28] T. Raïssi, G. Videau, A. Zolghadri, Interval observers design for consistency checks of nonlinear continuous-time systems, Automatica 46 (2010) 518-527.

[29] D. Efimov, L. Fridman, T. Raïssi, A. Zolghadri, R. Seydou, Interval es- 
timation for LPV systems applying high order sliding mode techniques, Automatica 48 (2012) 2365-2371.

[30] M. Blanke, M. Kinnaert, J. Lunze, M. Staroswiecki, Diagnosis and FaultTolerant Control, Springer-Verlag Berlin Heidelberg, 2006.

[31] C. Kunusch, P. Puleston, M. Mayosky, Sliding-Mode control of PEM fuel cells, Springer-Verlag, London, U.K., 2012.

[32] K. Tanaka, H. O. Wang, Fuzzy control systems design and analysis: A linear matrix inequality approach, John Wiley and Sons, Inc., 2001.

[33] S. K. Nguang, P. Shi, Robust $\mathcal{H}_{\infty}$ output feedback control design for fuzzy dynamic systems with quadratic $\mathcal{D}$ stability constraints: an lmi approach, Information Sciences 176 (2006).

[34] M. Chilali, P. Gahinet, $H_{\infty}$ design with pole placement constraints: an LMI approach, IEEE Transactions on Automatic Control 41 (1996) $358-367$.

[35] J. Meseguer, V. Puig, T. Escobet, J. Saludes, Observer gain effect in linear interval observer-based fault detection, Journal of Process Control 20 (2010) $944-956$. 
[36] J. F. Sturm, Using SeDuMi 1.02, a MATLAB toolbox for optimization over symmetric cones, Optimization methods and software 11-12 (1999) 625-653.

[37] J. Löfberg, YALMIP: A toolbox for modeling and optimization in MATLAB, in: Proceedings of the CACSD Conference.

[38] M. Chadli and H. R. Karimi, Robust observer design for unknown inputs Takagi-Sugeno models, IEEE Transactions on Fuzzy Systems 21 (2013) $158-164$.

[39] T. Escobet, D. Feroldi, S. de Lira, V. Puig, J. Quevedo, J. Riera, M. Serra, Model-based fault diagnosis in PEM fuel cell systems, Journal of Power Sources 192 (2009) 216-223.

[40] V. Puig, J. Quevedo, T. Escobet, F. Nejjari, S. de las Heras, Passive robust fault detection of dynamic processes using interval models, IEEE Transactions on Control Systems Technology 16 (2008) 1083-1089.

[41] V. Puig, J. Blesa, Limnimeter and rain gauge FDI in sewer networks using an interval parity equations based detection approach and an enhanced isolation scheme, Control Engineering Practice 21 (2013) 146 170. 
[42] S. Tornil-Sin, C. Ocampo-Martinez, V. Puig, T. Escobet, Robust fault diagnosis of nonlinear systems using interval constraint satisfaction and analytical redundancy relations, IEEE Transactions on System, Man and Cybernetics: Systems 44 (2014) 18-29.

[43] J. Blesa, D. Rotondo, V. Puig, F. Nejjari, FDI and FTC of wind turbines using the interval observer approach and virtual actuators/sensors, Control Engineering Practice 24 (2014) 138 - 155.

[44] P. A. N. Rosa, Multiple-model adaptive control of uncertain LPV systems, Ph.D. thesis, Instituto superior técnico, Universade Técnica de Lisboa, advised by C. Silvestre, 2011.

[45] M. W. Hirsch and H. L. Smith, Monotone maps: a review, Journal of Difference Equations and Applications 11 (2005) 379-398.

[46] J. Schur, Über Potenzreihen, die im Innern des Einheitskreises beschränkt sind, Journal für die reine und angewandte Mathematik 147 (1917) 205-232.

[47] Z.-P. Jiang, Y. Wang, Input-to-state stability for discrete-time nonlinear systems, Automatica 37 (2001) 857-869. 


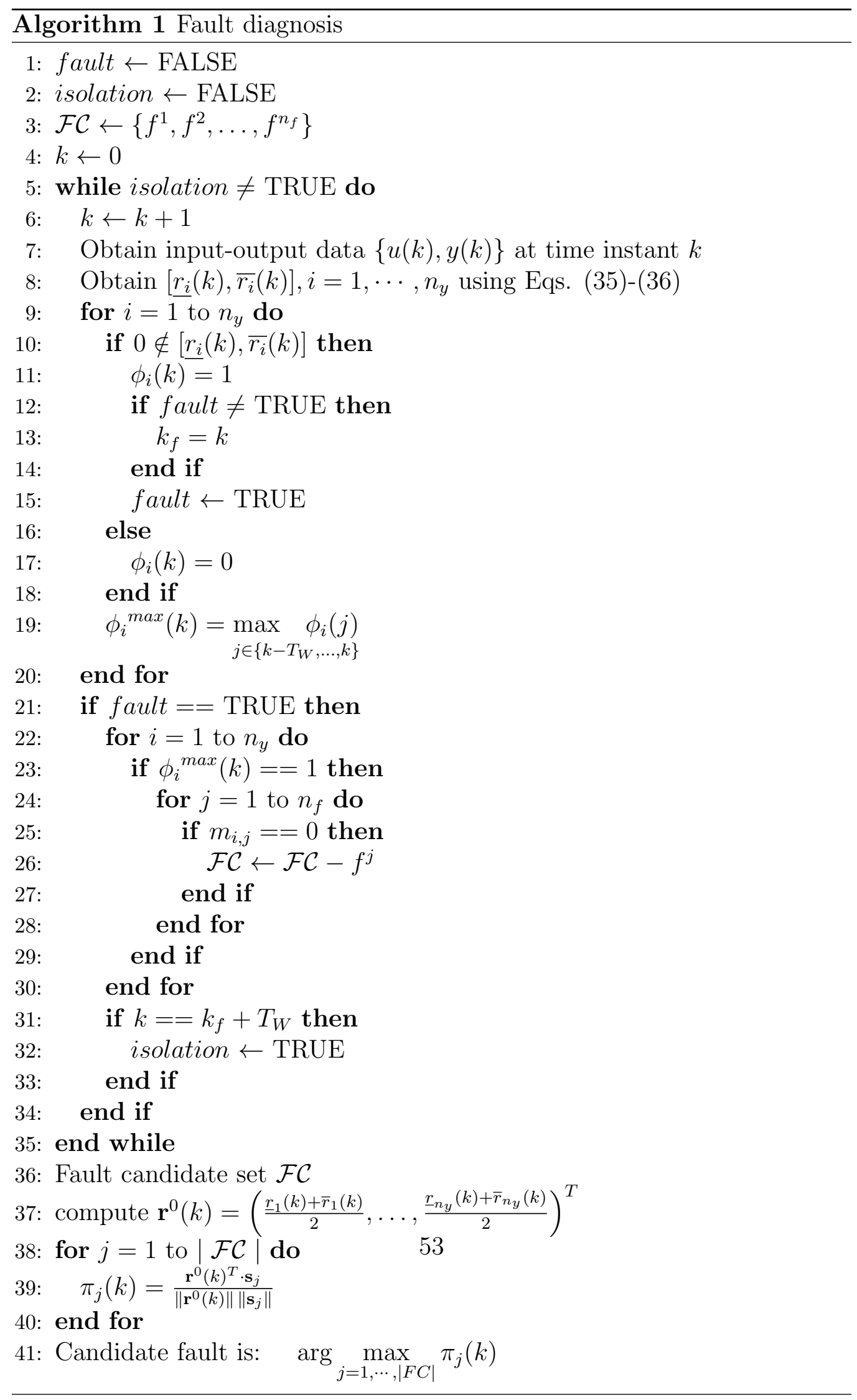

